\title{
Overall science goals and top level AO requirements for the E-ELT
}

\author{
Markus Kissler-Patig ${ }^{\mathrm{a}}$ \\ European Southern Observatory, Garching, Germany
}

Abstract. The E-ELT is the most ambitious of the ELTs that are planed. Its main characteristic is to be an adaptive telescope, i.e. AO is included in the telescope design. The science case for the E-ELT is largely driven by a diffraction limited image quality of the telescope. The science goals span from direct imaging and characterisation of exo-planets to studying the most distant objects in the universe. The science cases imply a long term deployment at the telescope of many AO flavours (ground-layer AO, single conjugated AO, laser tomography AO, multi-conjugated AO, multi-object AO, extreme $\mathrm{AO}$ ). While some of these are covered by the telescope, others will require post-focal $\mathrm{AO}$ modules feeding the instruments.

\section{Overview of the European Extremely Large Telescope (E-ELT)}

The European Extremely Large Telescope (E-ELT) project aims to provide European astronomers with the largest optical-infrared telescope in the World.

With a diameter of $42 \mathrm{~m}$ and being fully adaptive from the start, the E-ELT will be more than one hundred times more sensitive than the present-day largest optical telescopes. The E-ELT will vastly advance astrophysical knowledge by enabling detailed studies of planets around other stars, the first galaxies in the Universe, super-massive black holes, and the nature of the Universe's dark sector.

The E-ELT is being built by the European Southern Observatory on behalf on its 14 member countries. The project is currently in its detailed design phase, supplemented by a FP7 preparatory program under a grant from the European Commission. Decision to build is expected by late 2010, with a start of operation in 2018.

\footnotetext{
a e-mail: mkissler@eso.org
}

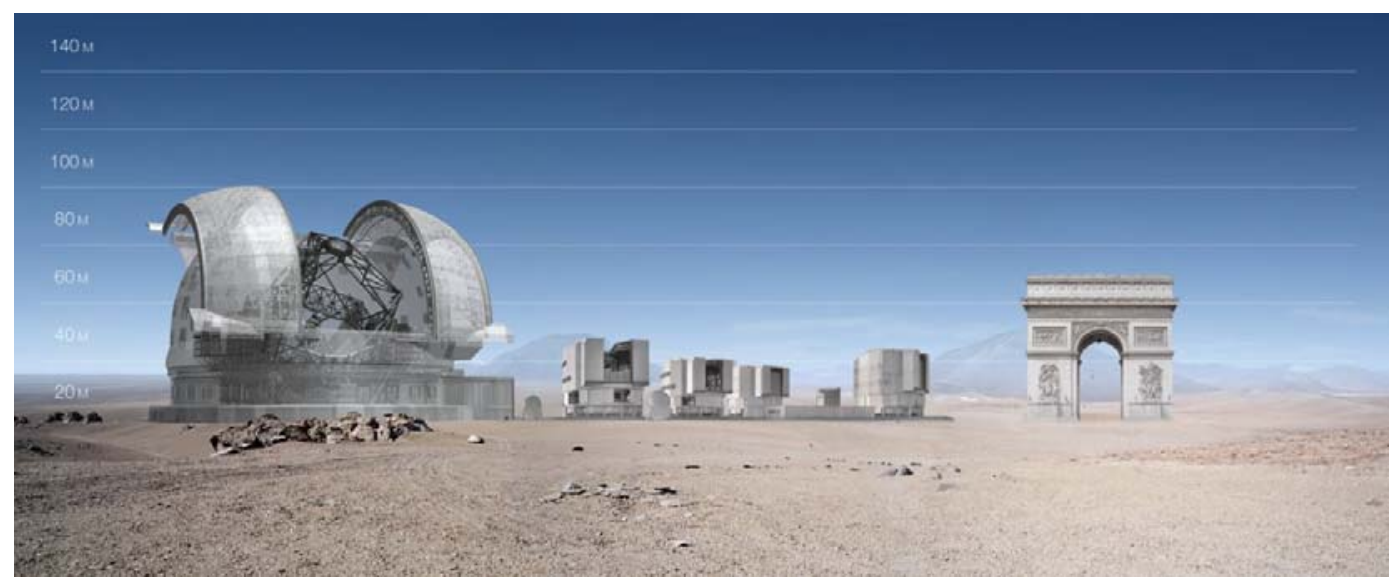

Fig. 1. The E-ELT, compared here with the VLT and Paris' Arc de Triomphe, will indeed be an extremely large telescope.

This is an Open Access article distributed under the terms of the Creative Commons Attribution-Noncommercial License, which permits unrestricted use, distribution, and reproduction in any noncommercial medium, provided the original work is properly cited. 

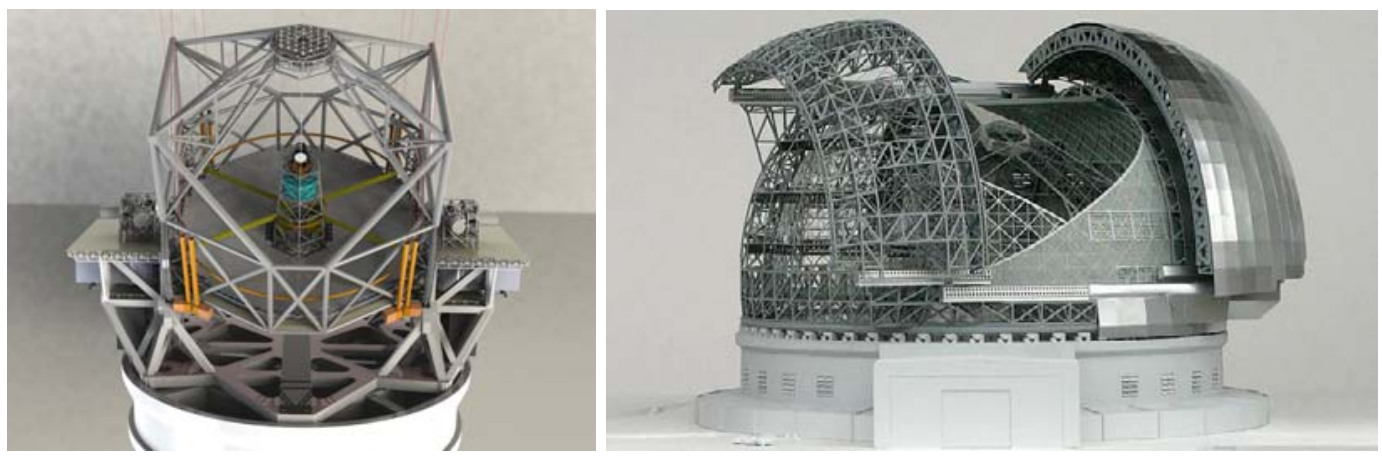

Fig. 2. Models of the E-ELT main structure (left) and the E-ELT dome (right).

\subsection{Telescope design}

The E-ELT is designed as a Nasmyth telescope (see Fig. 2). It has a footprint of about 80m diameter and is about $60 \mathrm{~m}$ high. The altitude and azimuth structures weigh together nearly 5000 tons. This structure supports the five mirror optical design and accomodates two Nasmyth platforms. Each platform is of about the size of a tennis court and can host several instruments. The E-ELT optical design differs from other telescope and ELT designs mostly by including adaptive optics into the telescope. This drove the optical layout to five mirrors (Sect. 1.2): a three mirror anastigmat with two flat folding mirrors providing the adaptive optics. This novel five-mirror design results in an exceptional image quality, with no significant aberrations in the 10 arcmin field of view.

Several designs were considered for the telescope enclosure. The project settled for a rather classical dome design, one model of which is shown in Fig. 2.

\subsection{Optical design}

The basic optical solution for the E-ELT is a folded three-mirror anastigmat, folding being provided by two flat mirrors sending the beams to either Nasmyth foci along the elevation axis of the telescope (Fig. 3). The primary mirror focal ratio is $\mathrm{f} / 1$. The total Nasmyth field of view is 10 arc minutes, limited by the dimensions of the way-through hole in one of the flat folding mirrors (M4). As-designed optical quality is diffraction limited up to the edge of the curved field at all wavelengths. Field curvature is low and approximately centred on the exit pupil. The Nasmyth focal ratio is rather large (f/17.7) and constrained by the backfocal distance, as well as by the location and size of mirrors M3 to M5.

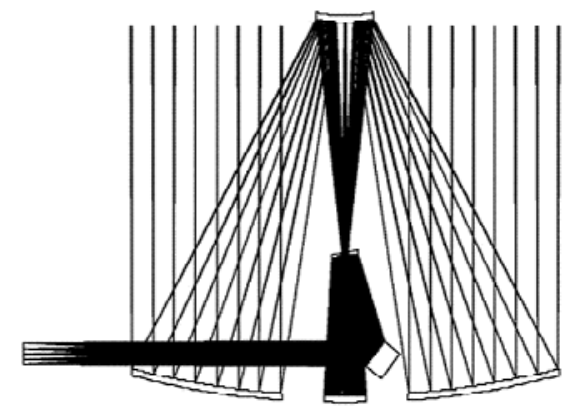

Fig. 3. E-ELT optical layout

The present concept features as a baseline a 42-m diameter mirror telescope. The primary mirror is composed of 984 hexagonal segments, each $1.45 \mathrm{~m}$ wide, while the secondary mirror is as large as 5.7 
$\mathrm{m}$ in diameter. The tertiary mirror, $4.0 \mathrm{~m}$ in diameter, relays the light to the adaptive optics system, which is composed of two mirrors: M4, a 2.6-m mirror supported by 5000 or more actuators so as to be able to distort its own shape a thousand times per second, and M5, $2.7 \mathrm{~m}$ in diameter, that allows for the final image corrections.

The folding arrangement (flat M4 and M5 mirrors) is conceived to provide conveniently located flat surfaces for an adaptive shell (M4, conjugated to a few hundred metres above the telescope) and field stabilisation (M5, with a minor off-pupil effect). It also allows a moderate tilt of the adaptive mirror. The design can be reconfigured to provide other foci besides the two Nasmyth ones: a Gravity-Invariant (after reflection on a 6th flat mirror) and an f/60 Coudé foci (with refractive image transport), with small field of view: 20 arc-seconds in diameter, limited by component size in the image transport.

An intermediate $\mathrm{f} / 4.15$ focus is also available (after reflection off the secondary mirror). Image quality is poor (mostly limited by coma) with a seeing-limited field of about 1 arc-minute. This focus is not conceived for science; it is collocated with the way-through hole in M4 and therefore inconvenient for instrumentation anyway. With some limitations it may, however, be used for mounting removable calibration sources for adaptive optics and/or for a general-purpose atmospheric dispersion compensator.

\section{Science at the diffraction limit}

The science case for the E-ELT builds on three pillars: a) contemporary science, represented by the science cases explored in the design reference mission, the design reference science plan and the instrument concept studies; b) synergies with other facilities such as the VLT, the JWST, ALMA, survey telescopes such as the LSST, the SKA and more; c) the discovery potential of the telescope, enabling the exploration of the unknown.

Most science cases rely at least on a partial correction of the image quality, many are inspired by the spatial resolution that a $42 \mathrm{~m}$ diameter telescope working at the diffraction limit offers.
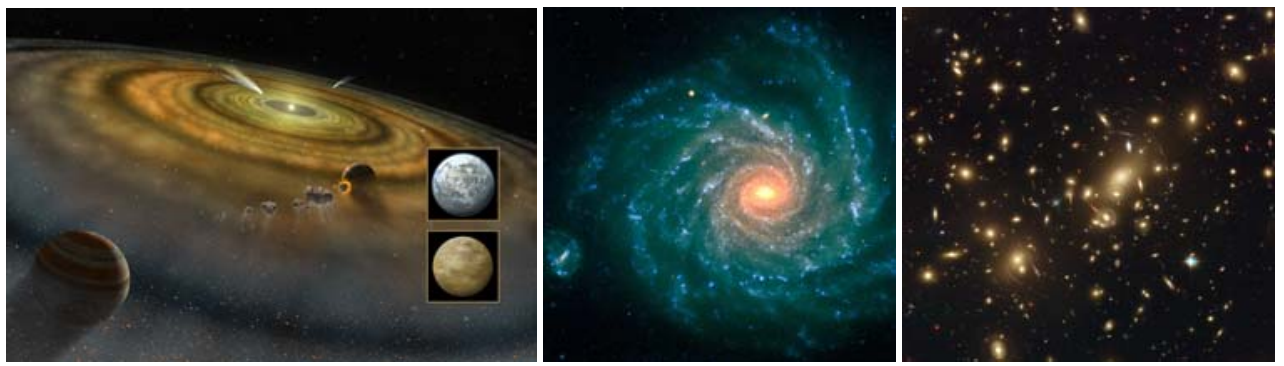

Fig. 4. The contemporary science drivers for the E-ELT include exo-planets, nearby stellar populations and distant galaxies.

\subsection{Contemporary science}

\subsubsection{Exo-planets}

The contemporary science includes the cases that can be extrapolated from today's research into the E-ELT era. Most prominently, it features the discovery and characterisation of exo-planets. This includes not only radial velocity searches for earth-mass planets, but also detection by astrometry, direct imaging and transit spectroscopy. The three latter methods rely on exquisite image quality. Astrometry, for example, will reach the levels of tens of micro-arcseconds per year when performed behind a 
diffraction limited imager fed by multi-conjugated adaptive optics. This is sufficient to detect Jupitermass (and potentially Neptune-mass) planets through the reflex motion of the parent star, in nearby systems. Such measurements would complement radial velocity measurements and remove the geometrical ambiguity inherent in the latter method.

Pushing the exo-planet research further, composition measurements of their atmospheres could become routine work. Presumably the easiest access to exo-planet atmospheres is provided through spectroscopy of planet-star systems during a primary transit of the planet in front of its star. During such events, the stellar light shines through the planet atmosphere. Through a differential measurement, the latter can be studied. This, however, requires a very high (on-axis) spatial resolution - which, though, is easily achievable as such a measurements always imply that a bright star (the planet host star) is on-axis.

Finally, exo-planet research will culminate with the direct detection of rocky planets in habitable zones. This measurement is arguably the most challenging one in this research field, as it requires contrast ratios of the order of a billion at a distance of a fraction of an arc-second of the bright guide star. Such performance is barely achievable with ELTs and certainly not without extreme adaptive optics. If successful though, it opens a new door to the characterisation of planets in habitable zones: certainly one of the ultimate goals for ELTs.

\subsubsection{Stars and Galaxies}

Among the other prominent cases, several rely on diffraction limited imaging: either on small fields, such as the study of circum-stellar and proto-planetary disks, or over fields of several arc-minutes, such as the study of the stellar/planetary mass functions and the observations of colour-magnitude diagrams of stellar populations in nearby galaxies.

In the two latter cases, the goal is to image point sources (stars/brown-dwarfs/jupiters) in very crowded fields. A comparison with the performance expected for JWST reveals that the size of the diffraction limited core of the PSF plays and even more important role than the Strehl ratio. Thus, such studies will profit immensely from multi-conjugated adaptive optics over fields of one to two arc-minutes, even at Strehl ratios of less than $50 \%$. Thanks for the E-ELT, astronomers will be able to understand the fragmentation of molecular clouds and the mass spectrum of stars and planets forming out of them. Further, colour-magnitude diagrams in nearby galaxies will reveal for the first time unambiguously the complicated composition of the stellar populations in these galaxies and thus the formation history of most of the stars in the universe.

\subsubsection{Distant Galaxies}

A further science goal in the contemporary category is the study of the highest redshift galaxies. This goal is also a top one for the JWST and the synergy of the facilities is particularly strong in that area. The role of the E-ELT will be to spatially resolve and study the distant galaxies. This requires, on the one hand, a good multiplex capability in order to beat the necessary long exposure times. On the other hand, the small sizes of distant galaxies call for a somewhat higher spatial resolution than provided by the seeing alone. Such corrections for image quality are needed punctually over a large field of view of several arc-minutes. Thus, multi-object adaptive optic is seen as an ideal match and would allow to study efficiently the formation of galaxies and structure in the early universe.

\subsection{Discovery space}

Synergies with other facilities are a very important component of the E-ELT science case, but will not be addressed further here. Instead, the importance of spatial resolution should be stressed for the discovery potential of the E-ELT. The E-ELT will have a spatial resolution 18 times higher than the HST, and still 7 times higher than the JWST (imagine observing at 3.5" seeing rather than 0.5"...). Clearly, the E-ELT will open a new area of the parameter space in this respect. Martin Harwit (2003) 
summarised nicely that opening parameter space, i.e. looking into areas where no one had a chance to look before, is the most important aspect driving discoveries.

The key point is that the E-ELT will approach the spatial resolution of near-infrared interferometers (such as the VLTI) but for sources nearly 20(!) magnitudes fainter. A spatial resolution of the order of 1 milli-arcsecond will be possible even for the faintest sources. In turn, this translates into astrometric measurements of tens of micro(!)-arcseconds for faint sources, and given a baseline of a few years, the ability to measure proper motions of the order of 10 micro-arcseconds per year!

At these spatial scales, it is close to impossible to predict what exciting science results await us.

In summary, all prominent science cases require some form or another of image quality correction. From simple ground-layer adaptive optics, to extreme adaptive optics, while passing through singleand multi-conjugated adaptive optics and multi-object adaptive optics (all with and without laser guide stars).

\section{Instrumentation and adaptive optics}

The construction proposal that will be delivered by the end of 2010 to the ESO council will include an instrumentation plan for the E-ELT.

In order to guide this plan, conceptual or phase A studies were launched in 2007/2008 for eight instruments and two adaptive optics modules. The summary of their properties are given in Table 1 (further information can be found on the project web pages).

Table 1. E-ELT instruments and AO modules currently undergoing conceptual or phase A studies. SCAO: Singleconjugated AO, MCAO: Multi-conjugated AO, MOAO: Multi-object AO, LTAO: Laser tomography AO, XAO: Extreme AO, IFU: integral-field unit (spectrograph), MOS: Multi-Object Spectrograph.

\begin{tabular}{|c|c|c|c|c|c|}
\hline Name & Instrument type & $\begin{array}{l}\text { Wavelength } \\
\text { range [microns] }\end{array}$ & $\begin{array}{l}\text { FoV and } \\
\text { sampling }\end{array}$ & $\begin{array}{l}\text { Spectral } \\
\text { resolution }\end{array}$ & AO support \\
\hline MICADO & $\begin{array}{l}\text { Diffraction limited } \\
\text { NIR Imager } \\
\text { (slit spectroscopy?) }\end{array}$ & $0.8-2.4$ & $\begin{array}{l}30 " \\
3-5 \text { mas/pix }\end{array}$ & $\sim 4000$ & $\begin{array}{l}\mathrm{SCAO} / \\
\mathrm{MCAO}\end{array}$ \\
\hline HARMONI & $\begin{array}{l}\text { Single-field } \\
\text { NIR spectrograph }\end{array}$ & $0.8-2.4$ & $\begin{array}{l}1 " \text { to } 10 " \\
4-40 \text { mas/pix }\end{array}$ & $\begin{array}{l}\sim 4000 \\
\sim 20.000\end{array}$ & $\begin{array}{l}\text { SCAO/ } \\
\text { LTAO }\end{array}$ \\
\hline EAGLE & $\begin{array}{l}\text { Wide-field, multi- } \\
\text { object NIR IFU }\end{array}$ & $0.8-2.4$ & $\begin{array}{l}\geq 5^{\prime} \\
\sim 40 \text { mas/pix }\end{array}$ & $\begin{array}{l}\sim 5000 \\
\sim 10.000\end{array}$ & MOAO \\
\hline CODEX & $\begin{array}{l}\text { High-R visual } \\
\text { spectrograph }\end{array}$ & $0.35-0.72$ & $\begin{array}{l}\text { point } \\
\text { source }\end{array}$ & 120.000 & Tip-tilt? \\
\hline METIS & $\begin{array}{l}\text { Mid-IR imager } \\
\text { and spectrograph }\end{array}$ & $3.5-20$ & $\begin{array}{l}30 " \\
15-30 \text { mas/pix }\end{array}$ & $\begin{array}{l}5-200 \\
\sim 100.000\end{array}$ & $\begin{array}{l}\text { SCAO/ } \\
\text { LTAO }\end{array}$ \\
\hline EPICS & Planet finder & $0.6-1.8$ & 2"-4" & $>50$ & XAO \\
\hline OPTIMOS & Optical MOS & $0.3-1.8$ & $5^{\prime}-10^{\prime}$ & 1000 or 10.000 & GLAO \\
\hline SIMPLE & $\begin{array}{l}\text { NIR high-R } \\
\text { spectrograph }\end{array}$ & $0.8-2.4$ & & $\sim 100.000$ & $\begin{array}{l}\text { SCAO/ } \\
\text { LTAO }\end{array}$ \\
\hline MAORY & $\begin{array}{l}\text { Multi-conjugated } \\
\text { AO module }\end{array}$ & $0.6-2.4$ & 2 ' & & $\begin{array}{l}2 \mathrm{DMs}+\mathrm{M} 4 \\
6 \mathrm{LGS}\end{array}$ \\
\hline ATLAS & $\begin{array}{l}\text { Laser tomography } \\
\text { AO module }\end{array}$ & $0.6-2.4$ & 1 ' & & $\begin{array}{l}\text { M4 } \\
6 \text { LGS }\end{array}$ \\
\hline
\end{tabular}

The need and potential for all flavours of adaptive optics as been recognised, and many variations of adaptive optics are included in the studies. These split into three broad categories: i) the adaptive optics modes included in the telescope, ii) the adaptive optics modes studied as stand-alone, posttelescope-focus modules, and iii) the adaptive optics modes studied as part of the instruments that they will serve. 
The first category, provided directly by the telescope, currently only includes ground-layer adaptive optics. In this mode, up to three natural guide stars, or up to four laser guide stars can be used to correct the inner 5' field of view of the telescope. This mode uses the wave-front sensors included in the telescope adapter/rotator, and the fourth (high-order) and fifth (fast tip-tilt) mirrors of the telescope. The second category includes laser tomography (single-conjugated) adaptive optics and multi-conjugated adaptive optics. Both are studied as stand-alone modules and provide their own wave-front sensors for six laser guide stars. Both use the fourth and fifth mirror of the telescope for corrections. In the case of the laser-tomography AO module, the telescope mirrors are the only deformable mirrors. In the case of the multi-conjugated $\mathrm{AO}$ module, the fourth telescope mirror, conjugated at a few hundred meters above the ground, is used to correct for ground-layer turbulence, while two further deformable mirrors inside the module are conjugated to about 5 and $12 \mathrm{~km}$. Both concept studies are designed without a specific instrument in mind and could be adapted to serve several of the studied instruments.

Finally, a few types of $\mathrm{AO}$ are so specialised, that it would not make sense to conduct studies that are decoupled from the instruments that they intend to serve. This is the case for extreme adaptive optics supporting the planet finder instrument, for the multi-object adaptive optics correcting the patrol field of the multi-integral field unit instrument, and for a potential flavour of single conjugated adaptive optics supporting the mid-infrared instrument. These types of AO are studied within the respective studies.

In summary, the detailed design phase of the E-ELT includes a large amount of AO studies, covering essentially all AO flavours potentially expected in the first decade. The studies will allow to evaluate the feasibility and challenges of each AO type and help developing the optimal implementation plan.

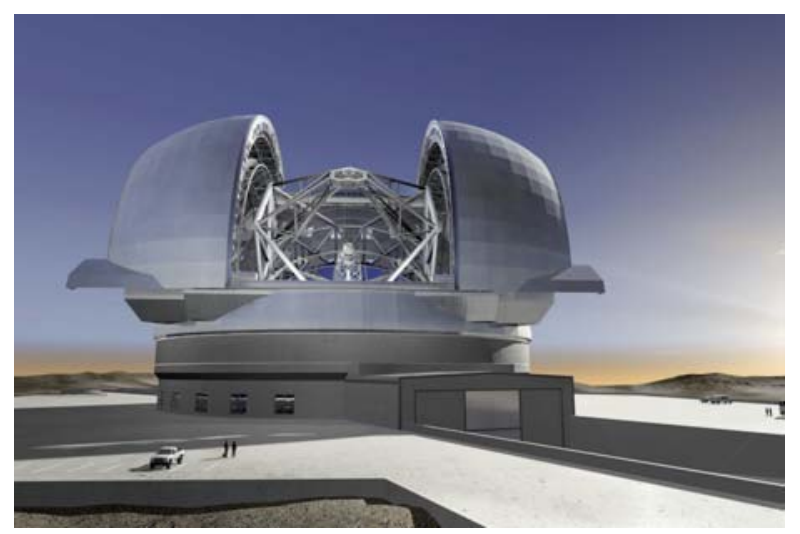

\section{Outlook}

The power of extremely large telescopes is provided by their large collecting area but even more so by their unprecedented spatial resolution.

The E-ELT in particular recognises this potential and presents many prominent science case that rely on improved image quality: from ground-based adaptive optics to extreme adaptive optics. Many flavours of adaptive optics are being studied in the course of the telescope's detail design phase. The greatest regret is that not all adaptive optics flavours can be implemented at first light. A wise implementation plan will have to be conceived, but surely the E-ELT will feature most of the adaptive optics technologies when reaching maturity.

\section{References}

1. Martin Harwit, Physics Today November issue, (2003) 38-43 